Benchmark of mul ti-phase method for the computat $i$ on of $f$ ast $i$ on di st $r i$ but $i$ ons i $n$ a tokanak pl asna in the presence of I ow ampl i t ude resonant MHD act i vi ty

\begin{tabular}{|l|l|}
\hline $\begin{array}{l}\text { j our nal or } \\
\text { publ } \mathrm{i} \text { cat } \mathrm{i} \text { on } \mathrm{ti} \mathrm{tl} \text { e }\end{array}$ & Comput er Physi cs Communi cat i ons \\
\hline vol une & 220 \\
\hline number & Novenber 2017 \\
\hline page range & $279-284$ \\
\hline year & $2017-08$ 09 \\
\hline URL & ht t p: //hdl . handl e. net /10655/00012820 \\
\hline
\end{tabular}




\title{
Benchmark of Multi-Phase Method for the Computation of Fast Ion Distributions in a Tokamak Plasma in the Presence of Low-Amplitude Resonant MHD Activity
}

\author{
A. Bierwage ${ }^{\mathrm{a}, *}$, Y. Todo ${ }^{\mathrm{b}}$ \\ ${ }^{a}$ National Institutes for Quantum and Radiological Science and Technology, Rokkasho Fusion Institute, Aomori 039-3212, Japan \\ ${ }^{b}$ National Institute for Fusion Science, 322-6 Oroshi-cho, Toki City, Gifu 509-5292, Japan
}

\begin{abstract}
The transport of fast ions in a beam-driven JT-60U tokamak plasma subject to resonant magnetohydrodynamic (MHD) mode activity is simulated using the so-called multi-phase method, where $4 \mathrm{~ms}$ intervals of classical Monte-Carlo simulations (without MHD) are interlaced with $1 \mathrm{~ms}$ intervals of hybrid simulations (with MHD). The multi-phase simulation results are compared to results obtained with a continuous hybrid simulations, which were recently validated against experimental data [A. Bierwage et al. Nucl. Fusion 57(1):0160036, 2017]. It is shown that the multi-phase method, in spite of causing significant overshoots in the MHD fluctuation amplitudes, accurately reproduces the frequencies and positions of the dominant resonant modes, as well as the spatial profile and velocity distribution of the fast ions, while consuming only a fraction of the computation time required by the continuous hybrid simulation. The present paper is limited to low-amplitude fluctuations consisting of a few long-wavelength modes that interact only weakly with each other. The success of this benchmark study paves the way for applying the multi-phase method to the simulation of Abrupt Large-amplitude Events (ALE), which were seen in the same JT-60U experiments but at larger time intervals. Possible implications for the construction of reduced models for fast ion transport are discussed.
\end{abstract}

Keywords: Multiple time-scale, Hybrid simulation, Energetic particles, Tokamak

\section{Introduction}

In order to enable simulations to accurately predict the confinement of fast ions and their effect on the bulk plasma (e.g., heating, current drive, torque) in magnetically confined fusion experiments, it is necessary to account for a variety of processes that cover a wide range of temporal scales. Magnetohydrodynamic (MHD) waves of the shear Alfvén branch, which can be excited by resonant interactions with fast ions, have oscillation periods on the $\mu$ s time scale. Individual instability pulses, including low-amplitude fluctuations as well as large relaxation events, grow and decay on the time scale of 10-100 $\mu \mathrm{s}$. On intermediate time scales of $0.1-10 \mathrm{~ms}$, these instabilities may cluster in bursts and exhibit frequency chirping. During their life time, these instabilities cause a redistribution (transport) of fast ions in phase space. This is particularly true for long-wavelength modes with toroidal mode numbers of

\footnotetext{
${ }^{*}$ Corresponding author

Email addresses: bierwage. andreas@qst.go.jp (A. Bierwage), todo@nifs.ac.jp (Y. Todo)

order $n \sim 1-10$, because they tend to saturate primarily as a result of radial fast ion transport, at amplitudes where MHD nonlinearities are still weak. This transport includes processes such as wave-particle trapping, phase mixing and orbit stochastization, which cause a flattening of gradients in the fast ion phase space density across the widths of the resonances $[1,2,3]$.

The gradients flattened by wave-particle interactions are restored by the effects of collisions and sources, such as fusion reactions, beam injection and radio frequency heating. Depending on the injected power and the size of the phase space volume that is relevant for a certain process, the effects of collisions and sources can become significant on short (sub-millisecond), long (seconds), or any intermediate time scale.

Owing to the growing performance of supercomputers, it has recently become possible to simulate all the above processes side-by-side in a self-consistent manner using so-called hybrid simulations, where the bulk plasma dynamics are described by an MHD model and the motion of fast ions is described by a gyrokinetic model using the particle-in-cell (PIC) method [4]. In that paper, we have simulated the dynamics of chirp- 
ing energetic particle modes (EPM [5]) with dominant toroidal mode number $n=1$, which were routinely observed in JT-60U tokamak experiments driven by a pair of powerful negative-ion-based neutral beams (N-NB). Since the full MHD model used in [4] includes fast magnetosonic waves, the time step size was $\Delta t_{\mathrm{mhd}} \approx 0.9 \mathrm{~ns}$. Thus, more than $10^{7}$ time steps were needed to simulate the $35 \mathrm{~ms}$ interval studied in [4]. Recently, that simulation has been continued, and results up to $60 \mathrm{~ms}$ are reported in the present paper.

These kinds of simulations are extremely expensive and even if run for several months, they are only barely able to cover the $100 \mathrm{~ms}$ time scale required to reproduce abrupt large-amplitude events (ALE [6]), which were also seen in the same JT-60U discharges and cause the fast ion density to fluctuate by as much as $20 \%$ $[7,8]$. This raises the question whether it is possible to speed the simulation up, if one is primarily interested in the form of the fast ion phase space distribution and not so much in the detailed dynamics of MHD modes.

The purpose of this paper is to show that the so-called multi-phase method [9] can be used to reduce the simulation time to a fraction of that required by the continuous hybrid simulation, while accurately reproducing the radial profile and velocity distribution of fast ions in an N-NB-driven JT-60U plasma. In Section 2, we describe the multi-phase method and evaluate its performance. Multi-phase and hybrid simulation results are presented an compared in Section 3, followed by a discussion and conclusions in Section 4. The word "hybrid" will be used to refer to the continuous hybrid simulation.

\section{Simulation method and performance}

We use the MHD-PIC hybrid code MEGA [10, 11, 12], which was recently extended with fast ion Larmor radius effects (via 4-point gyroaveraging) and realistic models for beam ion sources and collisions [4, 9, 13, 14]. In order to reduce the computation time needed to reach a steady state, where all processes - sources, collisions, MHD activity and thermalization losses - are in balance, the multi-phase method was introduced and applied to simulate fast ion dynamics in DIII-D tokamak experiments [9]. At least for low values $n \ll 10$ of the toroidal mode number, where the MHD model is most reliable, the multi-phase simulations were successfully validated against experimental measurements [13] and are now being applied to explore the physical mechanisms controlling fast ion confinement [14].

An overview of the multi-phase method applied to our N-NB-driven JT-60U scenario is shown in Fig. 1. As in $[9,13]$, we choose to interlace $4 \mathrm{~ms}$ periods of "classical" simulations (collisions and sources only, MHD off) with $1 \mathrm{~ms}$ periods of hybrid simulations (MHD, collisions and sources all included). In Fig. 1(a), one can see a burst of MHD fluctuations during each $1 \mathrm{~ms}$ hybrid phase. Figure 1(b) shows that the total stored fast ion energy increases steadily, so the MHDinduced global losses are ignorable. Locally, the $1 \mathrm{~ms}$ MHD bursts cause noticeable but small periodic fluctuations in the fast ion pressure profile, as can be seen in Fig. 1(c), where the time trace of the peak value of the fast ion beta profile $\operatorname{Max}\left\{\beta_{\mathrm{f}}(r)\right\}$ is plotted. Here, $\beta_{\mathrm{f}}=2 \mu_{0} P_{\mathrm{f}} / B_{0}^{2}$ measures the ratio of kinetic pressure, $P_{\mathrm{f}}=\int \mathrm{d}^{3} v \mathcal{J}_{\mathrm{gc}} F_{\mathrm{f}} E$, to magnetic pressure, $B_{0}^{2} /\left(2 \mu_{0}\right)$, where $\mu_{0}$ is the vacuum permittivity, $B_{0}$ the magnetic field strength at the plasma center (magnetic axis), $F_{\mathrm{f}}$ is the fast ion guiding center phase space density, $\mathcal{J}_{\mathrm{gc}}$ is the guiding center Jacobian for the chosen set of coordinates, and $E=m_{\mathrm{D}} v^{2} / 2$ is the kinetic energy of a deuteron with mass $m_{\mathrm{D}}$ and velocity $v$.

The duration of the MHD off/on periods $(4+1 \mathrm{~ms})$ was chosen empirically based on the following considerations. The interval of $5 \mathrm{~ms}$ corresponds to the lower bound of the $5-10 \mathrm{~ms}$ periods at which fast-ion-driven instability bursts were typically seen in JT-60U experiments. The $1 \mathrm{~ms}$ duration for the hybrid phase (with MHD) was chosen because this is the typical time scale for a fast-ion-driven instabilities to grow to a significant amplitude, saturate and decay. Here, the attribute "significant" means that the instability is able to flatten the fast ion phase space gradients to such a degree that a mode with similar frequency, mode structure and magnitude is unlikely to occur within the next $4 \mathrm{~ms}$.

For the DIII-D tokamak scenario studied in [9], it has been shown that increasing the $5 \mathrm{~ms}$ intervals $(4+1 \mathrm{~ms})$ to $10 \mathrm{~ms}$ intervals $(9+1 \mathrm{~ms})$ had no significant effect on the simulation results. Both multi-phase simulations gave very similar steady-state fast ion pressure profiles and MHD fluctuation levels [9].

Here, we report the first comparison between a " $4+$ $1 \mathrm{~ms}$ " multi-phase simulation and a hybrid simulation with continuous MHD activity, using the JT-60U scenario in Fig. 1. The simulation parameters are summarized in Table 1. Note that wave-particle interactions in the hybrid simulation were simulated only for the $n=1-3$ harmonics, because $n=4$ was found to play no role in the present case, except for (weak) nonlinear MHD damping. See [4] for further details and a list of physical parameters.

We focus on the first $60 \mathrm{~ms}$ after the start of beam injection. On the Helios supercomputer at IFERC in Rokkasho/Japan, which was in operation until the end 


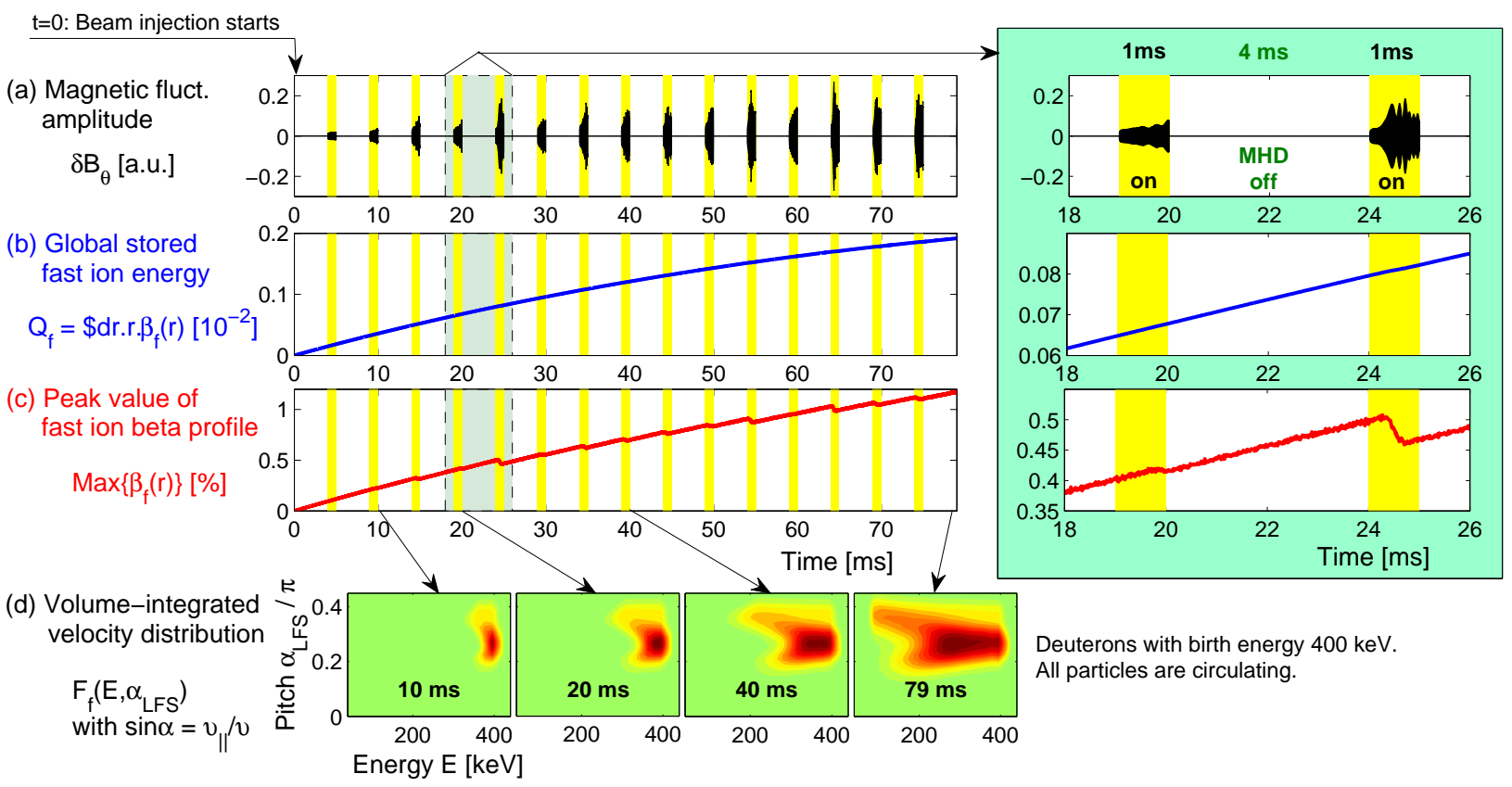

Figure 1: Overview of a multi-phase simulation for the beam-driven JT-60U plasma previously studied in [4]. Starting at $t=0$, a pair of tangential N-NBs continuously injects ions with a kinetic energy of $400 \mathrm{keV}$. Collisions with the thermal bulk plasma are also simulated continuously as described in [4]. At the end of each $5 \mathrm{~ms}$ interval, MHD fluctuations and their interactions with the fast ions are simulated for $1 \mathrm{~ms}$ (yellow shaded stripes). Time traces are shown for (a) the fluctuation amplitude of the poloidal magnetic field $\delta B_{\theta}(t)$ measured in the region where the $n=1$ mode peaks, (b) the global stored energy defined as the radial integral of the fast ion beta profile $Q_{\mathrm{f}}=\int \mathrm{d} r r \beta_{\mathrm{f}}(r)$, and (c) the peak value of the fast ion beta $\operatorname{Max}\left\{\beta_{\mathrm{f}}(r)\right\}$. The box on the right-hand side shows enlarged a time window of $8 \mathrm{~ms}$. Snapshots of the volume-integrated fast ion velocity distribution $F_{\mathrm{f}}\left(E, \alpha_{\mathrm{LFS}}\right)$ are shown in (d) using the pitch angle $\alpha=\arcsin \left(v_{\|} / v\right)$ evaluated on the low-field side ("LFS") of the midplane [15].

\begin{tabular}{|l|c|c|}
\hline & Hybrid & Multi-phase \\
\hline \hline Spatial grid & $\left(N_{R}, N_{\varphi}, N_{Z}\right)=(384,96,352)$ \\
\hline MPI domains & \multicolumn{2}{|c|}{$\left(M_{R}, M_{\varphi}, M_{Z}\right)=(16,16,16)$} \\
\hline MHD harmonics & \multicolumn{2}{|c|}{$n_{\text {mhd }}=0-4$} \\
\hline Driven harmonics & $n_{\text {drv }}=1-3$ & $n_{\text {drv }}=1-4$ \\
\hline
\end{tabular}

Table 1: Numerical parameters. The values for the spatial grid and MPI domains refer to right-handed cylinder coordinates $(R, \varphi, Z)$ Toroidal harmonics other than the $n_{\text {mhd }}$ values listed in the table are filtered out after each MHD time step $\Delta t_{\mathrm{mhd}}$. The values for $n_{\mathrm{drv}}$ are the toroidal harmonics that were retained in the effective fast ion current density $\boldsymbol{j}_{\mathrm{f} \text {,eff }}$, which is responsible for wave-particle interactions in the MHD momentum balance equation (Eq. (3) in [4]). Information about time steps and particle injection rates is given in the text.

of 2016 [16], the continuous hybrid simulation of this $60 \mathrm{~ms}$ interval took 3 full months, with the performance decreasing over time as the number of simulation particles increased. The ratio of injection time step to particle pushing time step was $\Delta t_{\text {inj }} / \Delta t_{\text {push }}=1$, and we performed MHD subcycling with $\Delta t_{\mathrm{push}} / \Delta t_{\mathrm{mhd}}=4$. As a result, 17.6 $\mathrm{M}$ simulation particles were injected during the course of the continuous hybrid simulation. About 17.0 M were still confined at $t=60 \mathrm{~ms}$, which means that only $3.5 \%$ of the injected particles hit the wall or are lost by thermalization ${ }^{1}$ some time during the simulated $60 \mathrm{~ms}$ period.

The multi-phase simulation in Fig. 1 was performed with a larger ratio $\Delta t_{\text {inj }} / \Delta t_{\text {push }}=4$, so that only $4.4 \mathrm{M}$ particles were injected during the simulated $60 \mathrm{~ms}$. As can be seen in Fig. 2(a), the number of confined particles at that time is $4.2 \mathrm{M}$, so the loss fraction (4.5\%) is still negligible, although somewhat larger than in the continuous hybrid simulation, presumably because the fluctuation amplitudes tend to overshoot each time the MHD solver is turned on (cf. Fig. 3 below).

Our choice to perform the continuous hybrid simulation with a 4 times larger number of simulation particles was motivated by the speculation that it could be more sensitive to PIC noise than a comparable multi-phase

\footnotetext{
${ }^{1}$ Thermalization loss means that a simulation particle is discarded when its energy falls below a certain threshold. Here, this threshold is chosen to be $80 \mathrm{keV}$, which corresponds to the energy of positive-ionbased neutral beams (P-NB) in JT-60U, which are not included in the present simulation. The first thermalization losses occur after about $43.6 \mathrm{~ms}$, mainly in the cooler and more collisional peripheral plasma. However, even at $t=60 \mathrm{~ms}$, the number of particles lost in this way is still negligibly small.
} 

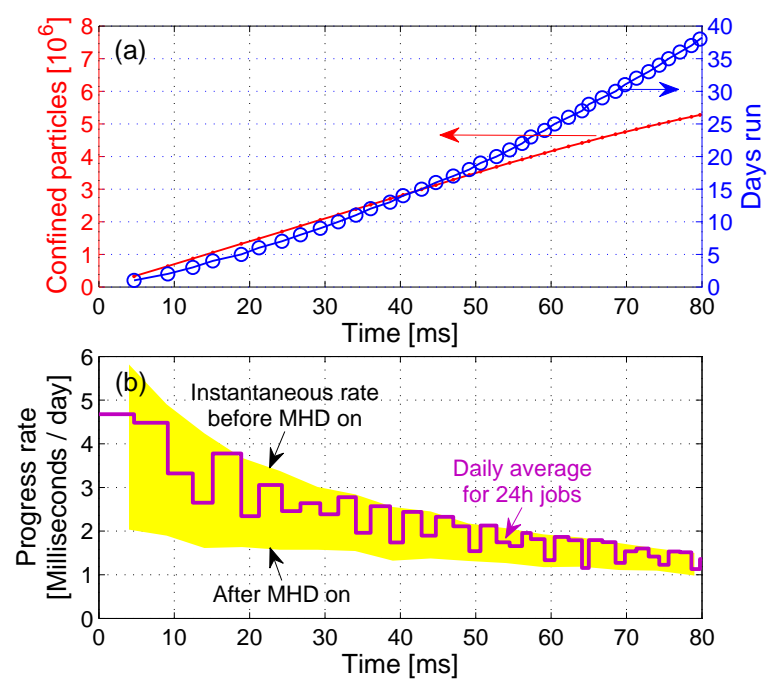

Figure 2: Performance of a " $4+1$ ms" multi-phase simulation for a beam-driven JT-60U plasma performed on the Helios supercomputer. (a): Number of confined simulation particles (= number of injected minus number of lost particles) as a function of simulated physical time, and the number of days the simulation has been running. (b): Progress rate (ms/day) at which the simulation advanced. The upper and lower bounds of the shaded area indicate the instantaneous progress rate measured just before (upper) and just after (lower) the MHD module is turned on. The daily average progress rate is shown as a stepped curve. Note that, from about $t=60 \mathrm{~ms}$ onward, the simulation of an $1 \mathrm{~ms}$ hybrid phase (with MHD) takes more than 24 hours. On those days, the daily average of the progress rate drops near the lower bound of the shaded area.

simulation. Conversely speaking, we are assuming that the multi-phase simulation may be less sensitive to PIC noise because of two reasons: the overshooting MHD fluctuation amplitudes yield a larger signal-to-noise ratio, and there is a smaller risk of accumulating effects of spurious signals, since the fluctuating fields are reset periodically. In the future, it may be worth to investigate this idea further. If multi-phase simulations can indeed be shown to be less sensitive to PIC noise, the reduction of the number of simulation particles could be used to further enhance the performance of the method.

In Fig. 2(a) one can see that, with the present setup, the multi-phase simulation needed only 25 days to simulate the $60 \mathrm{~ms}$ that took 93 days with the hybrid simulation. Of course, the reduced number of simulation particles contributed to this speed-up. However, by comparing the upper and lower limits of the shaded area in Fig. 2(b), one can see that until about $25 \mathrm{~ms}$, most of the computation time is consumed by the MHD solver, whose time step size is $1 / 4$ of that of the particle pusher. At $t=25 \mathrm{~ms}$ ( $1.7 \mathrm{M}$ particles) about $50 \%$ of the time is spent with MHD calculations, and at $t=80 \mathrm{~ms}(5.3$ $\mathrm{M}$ particles) it is about $30 \%$. Clearly, the merit of using the multi-phase method becomes smaller as time goes by and the number of particles increases, until the thermalization and wall losses balance the particle injection rate.

Note that one reason for the rapid drop of the multiphase simulation performance is the gyroaveraging, which is physically essential in the present case [17] but makes the PIC calculation nonlocal. The Larmor radius of the $400 \mathrm{keV}$ ions in the present low-field JT-60U plasma with $B_{0}=1.2 \mathrm{~T}$ is about $7.6 \mathrm{~cm}$, while the spatial extent of an MPI domain is about $14-20 \mathrm{~cm}$. Thus, there is a substantial number of guiding centers that require communication between neighboring MPI domains to perform the gyroaverage. Roughly speaking, when the number of simulation particles contained in an MPI domain exceeds the number of grid cells in the poloidal $(R, Z)$ plane (here $384 / 16 \times 96 / 16 \times 352 / 16=$ 3168 ), it may become worth to communicate the field data from entire neighboring MPI domains (not done here) instead of communicating individual satellite particle coordinates (as is done here). However, since the particle distribution is highly nonuniform in space, it is not straightforward to determine the threshold in the particle number beyond which the communication of fields becomes more efficient than communicating satellite particles. Moreover, there are constraints imposed by the amount of memory available on each CPU, and it is likely that the threshold also depends on the machine architecture, so needs to be measured case-by-case.

\section{Results}

The increased performance of the multi-phase simulation compared to the continuous hybrid simulation comes at the expense of generating a different history of dynamics. Simply speaking, during $1 \mathrm{~ms}$ of the " $4+1$ ms" multi-phase simulation the MHD fluctuations must, at least on average, cause the same amount of fast ion transport as the continuous MHD fluctuations in the continuous hybrid simulation do in $5 \mathrm{~ms}$.

Clearly, this is possible only if the fluctuation amplitudes are larger in the multi-phase simulation, which can indeed be observed in Fig. 3. Note in Fig. 3(b) that the fluctuation amplitudes in the multi-phase simulation tend to become large only every $10 \mathrm{~ms}$. This suggests that similar results may be obtained if the classical phase is extended to $9 \mathrm{~ms}$ as in [9].

Figure 3 also shows that the fluctuations with toroidal mode number $n=1$ dominate at all times, both in the multi-phase and in the hybrid simulation. However, from $t \gtrsim 35 \mathrm{~ms}$ onward, the amplitude of the $n=2$ harmonic often comes close to that of $n=1$. This is due 


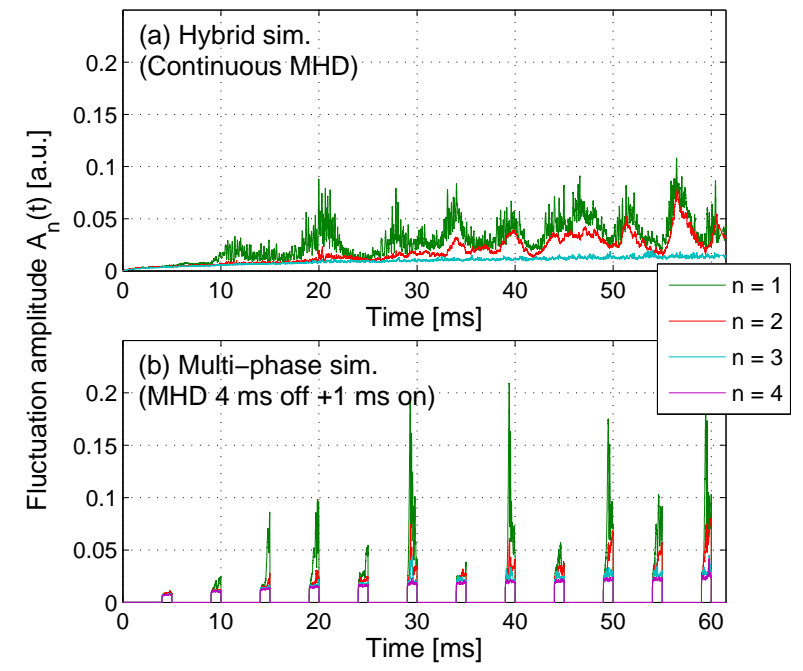

Figure 3: Comparison of the traces $A_{n}(t)=W_{n}^{1 / 2}(t)$ of the fluctuation amplitudes for toroidial mode numbers $n=1-4$ in (a) the continuous hybrid and (b) the multi-phase simulation. Here $W_{n}=$ $\frac{1}{2} \int \mathrm{d}^{3} x\left(\left|\rho_{\mathrm{b}}^{1 / 2} \delta \boldsymbol{u}\right|_{n}^{2}+|\delta \boldsymbol{B}|_{n}^{2}\right)$ is the volume-integrated MHD fluctuation energy for $|n|>1$, computed from the bulk ion mass density $\rho_{\mathrm{b}}$, and the fluctuating components $\delta \boldsymbol{u}$ and $\delta \boldsymbol{B}$ of the single-fluid MHD velocity and magnetic field vectors.

to the fact that the radial transport caused by $n=1$ together with collisional slowdown fill up the phase space domain on which the $n=2$ resonances feed [15]. Meanwhile, the amplitudes of the $n=3$ and $n=4$ harmonics are still negligibly small, in part owing to the inclusion of gyroaveraging effects, which effectively reduce the drive on higher- $n$ modes and prevents these harmonics from growing to unrealistically large amplitudes [17].

As was shown in detail in [4] and in preceding studies, the dominant $n=1$ harmonic exhibits up- and downward chirping in the $30-70 \mathrm{kHz}$ range, both in experiments and simulations. In Fig. 4(a), one can see that the $n=1$ signal in the multi-phase simulation performs similar chirps in precisely the same frequency band (40$60 \mathrm{kHz}$ ) as in the hybrid simulation. Moreover, Fig. 4(b) shows that the peak locations of the dominant $n=1$ wave packets in the multi-phase and hybrid simulations also agree and exhibit similar in- and outward propagation in the radial interval $0.4 \lesssim r / a \lesssim 0.48$.

Figure 5 shows that similarly good agreement is obtained for the $n=2$ harmonic. Although not visible here, the $n=2$ fluctuations during the first half of the simulation $(t \lesssim 35 \mathrm{~ms})$ are actually dominated by nonlinear MHD distortions of the dominant $n=1$ harmonic, so they oscillate at about twice the $n=1$ frequency of about $50 \mathrm{kHz}$; i.e., around $100 \mathrm{kHz}$ (beyond the range shown in Fig. 5(a)). During the second
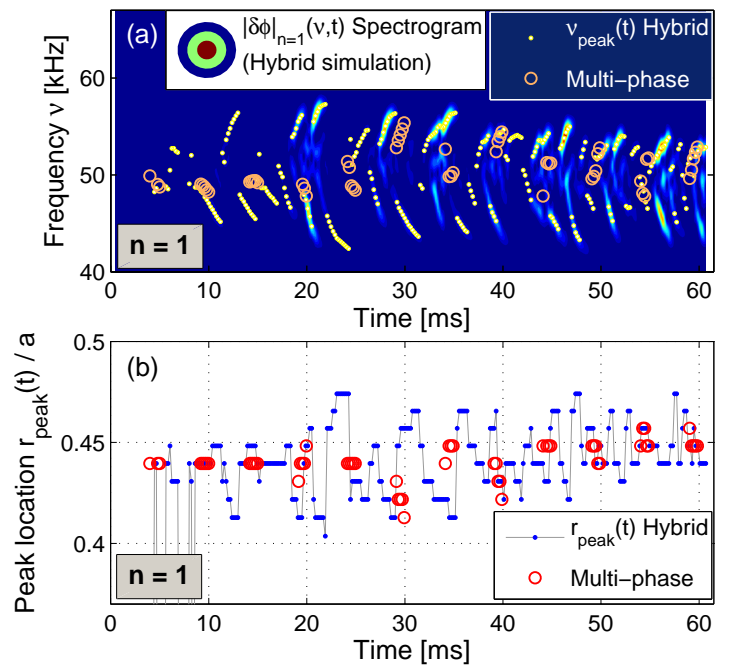

Figure 4: Comparison of the frequency and radial location of the dominant $n=1$ harmonic in the continuous hybrid and multi-phase simulations. For the hybrid simulation, panel (a) shows the spectrogram of the electrostatic potential fluctuations $|\delta \phi|_{n=1}(v, t)$ as a colored contour plot, where the frequency $v$ is not to be confused with the velocity $v$. The frequency $v_{\text {peak }}(t)$ at which the spectrogram peaks at a given time is indicated by dots (hybrid) and circles (multi-phase). The corresponding radial locations $r_{\text {peak }}(t)$ at which the spectrograms peak at each time are plotted in (b). The size of the time window used for the Fourier analysis is $\Delta t_{\text {win }}=0.5 \mathrm{~ms}$. See [4] for further details about the spectral analysis method used.

half of the simulation ( $t \gtrsim 35 \mathrm{~ms}$ ), the $n=2$ fluctuations are dominated by independent resonantly-driven modes, whose total fluctuation energy is comparable to that of $n=1$, as we have seen in Fig. 3 above. However, Fig. 5 shows that these resonant $n=2$ modes have lower frequencies and are located at different radii than the $n=1$ fluctuations. Although a detailed analysis of phase space islands remains to be carried out, it seems that the $n=1$ and $n=2$ modes interact only weakly or not at all via their respective resonant particles, so we believe that resonance overlaps do not play a significant role during the $60 \mathrm{~ms}$ time interval simulated here.

These resonantly excited shear Alfvén waves cause a redistribution of fast ions; especially in the radial direction. As was already mentioned in Section 2 above, only a very small fraction of the particles travels far enough for their orbits to intercept the wall. A global measure of the confined fast ion population is the stored energy, which we denote as $Q_{\mathrm{f}}$ and compute by spatially integrating the fast ion beta profile as $Q_{\mathrm{f}}=\int \mathrm{d} r r \beta_{\mathrm{f}}(r)$. Its absolute value in the multi-phase simulation was shown in Fig. 1(b) above. In Fig. 6(a), one can see that $Q_{\mathrm{f}}$ value in the multi-phase simulation deviates from that in the continuous hybrid simulation by only $0.2 \%$ or less. 

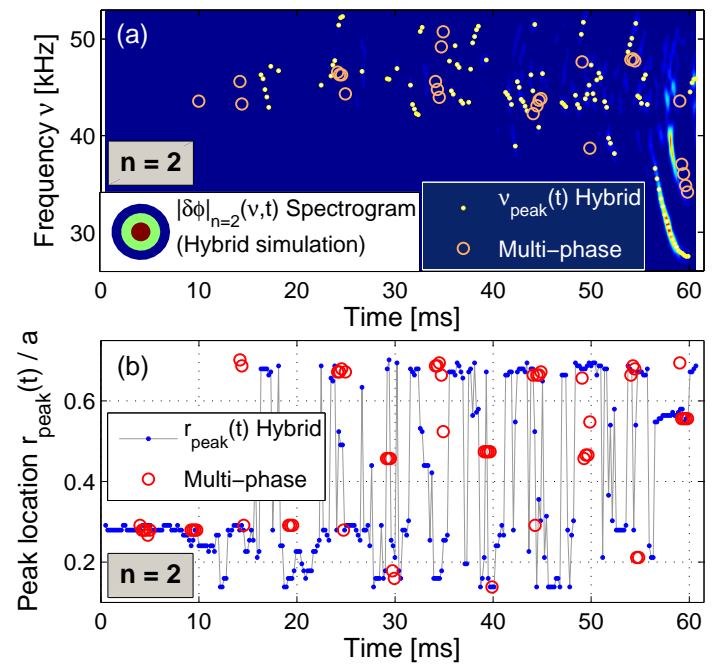

Figure 5: Comparison of the frequency and radial location of the second dominant $n=2$ harmonic in the continuous hybrid and multiphase simulations. Arranged as Fig. 4.

The small fluctuations of $Q_{\mathrm{f}}(t)$ that can be seen in Fig. 6(a) are correlated with the onset of MHD bursts in the multi-phase simulation and, to a lesser degree, also in the continuous hybrid simulation. Abrupt fluctuations on the $0.1 \mathrm{~ms}$ time scale are thought to be caused by prompt losses that occur when fast ions hit the wall. In addition, $Q_{\mathrm{f}}$ fluctuations on the time scale of a few $\mathrm{ms}$ or longer may be attributed to variations in the velocity distribution. This is because $\beta_{\mathrm{f}}$ used to calculate $Q_{\mathrm{f}}$ is a moment of the fast ion phase space density weighted by their kinetic energy $E$. Due to the fact that the collisional drag increases with the radially decreasing electron temperature $T_{\mathrm{e}}(r)$ (cf. Fig. 3 in [4]), the differences in the timing and amount of radial fast ion transport in the hybrid and multi-phase simulation ultimately leads to differences in the velocity distribution and, thus, in the evolution of $Q_{\mathrm{f}}$.

As a more local measure of the confined fast ion population, Fig. 6(b) shows the evolution of the peak value $\operatorname{Max}\left\{\beta_{\mathrm{f}}(r)\right\}$ of the fast ion beta profile. One can see excellent agreement between the hybrid and multi-phase simulation results, which both lie below the value obtained with a purely "classical" Monte-Carlo simulation, which includes only sources, collisions and losses in a static axisymmetric magnetic field, without any MHD activity.

Finally, Fig. 7 shows the detailed form of the instantaneous fast ion phase space distribution at $t=60 \mathrm{~ms}$, using three different projections. One can see that the radial profiles as well as the velocity distribution agree
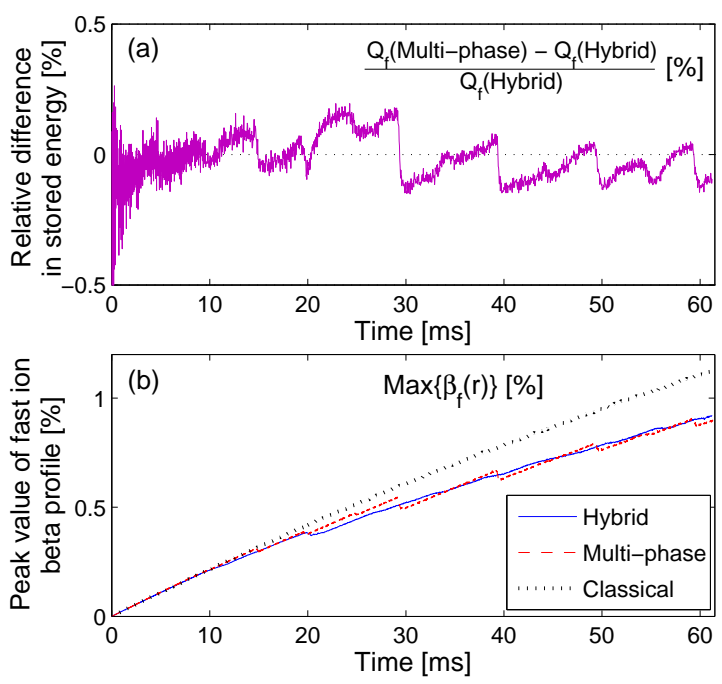

Figure 6: Comparison of temporal evolution of the fast ion population in hybrid, multi-phase and "classical" (no MHD) simulations. (a): Relative difference $\left[Q_{\mathrm{f}}\right.$ (Multi-phase $)-Q_{\mathrm{f}}$ (Hybrid) $] / Q_{\mathrm{f}}$ (Hybrid) of the stored fast ion energy $Q_{\mathrm{f}}(t)$ in the multi-phase and continuous hybrid simulations. (b): Peak value $\operatorname{Max}\left\{\beta_{\mathrm{f}}(r)\right\}$ of the fast ion beta profile.

very well. Since the snapshot was taken at the end of the interval $59 \mathrm{~ms} \leq t \leq 60 \mathrm{~ms}$ where MHD activity was simulated in the multi-phase simulation, the fast ion beta in the inner core region $0 \lesssim r / a \lesssim 0.3$ is slightly lower than in the continuous hybrid simulation. At $t=59 \mathrm{~ms}$, just before the MHD phase, it was slightly higher by about the same amount (not shown here).

\section{Discussion and conclusion}

In this paper, we have demonstrated that the so-called multi-phase method [9] accurately predicts the redistribution of fast ions in the presence of MHD fluctuations (here, shear Alfvén waves), which are resonantly excited by the fast ions themselves. In contrast to an earlier study [9], where the results of two multi-phase simulations were compared, the results of a continuous hybrid simulation served as a benchmark here.

As a working example, we considered a beamdriven JT-60U plasma and constrained our analysis to a time window where only low-amplitude activity of long-wavelength energetic particle modes (EPM) was present. The multi-phase simulation accurately reproduced the frequency and radial location of the dominant modes, which have toroidal mode numbers $n=1$ and $n=2$. The main difference between the hybrid and multi-phase simulation is that the MHD fluctuations in the latter case tend to overshoot, reaching more than 2 times higher amplitudes. 

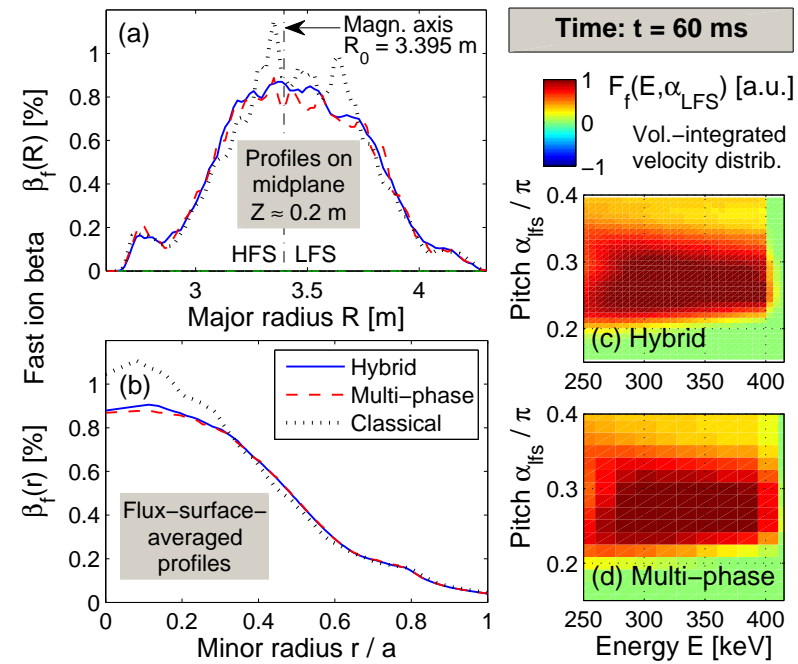

Figure 7: Comparison of the fast ion distributions at $t=60 \mathrm{~ms}$ in the hybrid, multi-phase and "classical" (no MHD) simulations. (a): Cross-section of the fast ion beta field $\beta_{\mathrm{f}}(R, Z)$ evaluated at $Z \approx 0.2 \mathrm{~m}$; i.e., near the midplane of the plasma whose magnetic axis is located at $\left(R_{0}, Z_{0}\right)=(3.395 \mathrm{~m}, 0.204 \mathrm{~m})$. The high-field side (HFS) and lowfield side (LFS) are indicated. (b) Flux-surface-averaged minor radial profiles $\beta_{\mathrm{f}}(r)$ of the fast ion beta. Note that, although the simulations were performed with gyroaveraging, the profiles in (a) and (b) were computed from the guiding center positions. (c) and (d): Color contour plots of the volume-integrated velocity distributions $F_{\mathrm{f}}\left(E, \alpha_{\mathrm{lfs}}\right)$ in the hybrid and multi-phase simulations. Note that the diagnostic mesh used for the velocity space in the hybrid simulation (c) has a 2 times higher resolution in each coordinate than in the multi-phase simulation (d).

The fast ion distributions were shown to agree well, in spite of the large difference in the mode amplitudes. This fact may have important implications for ongoing efforts to construct reduced models for fast ion transport. Namely, it is possible that the overall fast ion transport is insensitive to the detailed time history of the MHD fluctuations, which may occur in short pulses with large amplitudes (as in our multi-phase simulation) or in the form of longer burst with lower amplitudes (as in our hybrid simulation). It must be emphasized however, that this situation may change significantly when multiple modes grow to large amplitudes and nonlinear resonance overlaps occur, which did not seem to happen during the $60 \mathrm{~ms}$ time interval studied here.

The cumulative fast ion losses constituted a small fraction $(3.5 \%)$ of the total number of particles injected over the simulated $60 \mathrm{~ms}$. In the multi-phase simulation, the loss was larger ( $4.5 \%)$, presumably due to the abovementioned overshoots of the MHD fluctuation amplitude. This $30 \%$ increase in the number of lost particles suggests that multi-phase simulations may not be suitable for accurate predictions of wall heat loads. Of course, the accuracy of the losses predicted by the continuous hybrid simulation also remains to be checked.

The flux-surface-averaged fast ion beta profiles $\beta_{\mathrm{f}}(r)$ in Fig. 7(b) as well as the time traces of $\operatorname{Max}\left\{\beta_{\mathrm{f}}(r)\right\}$ in Fig. 6(b) give the impression that MHD activity causes a significant reduction of the fast ion beta value by up to $20 \%$ in the central core region $0 \lesssim r / a \lesssim 0.3$. However, it turns out that this is somewhat misleading. The midplane cross-section $\beta_{\mathrm{f}}(R)$ of the fast ion beta field in Fig. 7(a) shows that the difference between the beta profiles obtained without (classical) and with MHD (hybrid and multi-phase) is mostly due to narrow peaked structures near the plasma center. These spikes originate from the nonuniformity of the fast ion deposition (see also Fig. 4 in [4]) and are smoothed out by the MHD fluctuations. Except for these spikes, the profiles are actually rather similar to the classical prediction.

Therefore, instead of carrying out relatively expensive multi-phase or even more expensive continuous hybrid simulations, one may try to simply post-process the classical result with an empirical spatial smoothing algorithm. In this way, one may be able obtain a fairly accurate prediction for the fast ion beta field $\beta_{\mathrm{f}}(R, Z)$ at a small fraction of the computational cost, ${ }^{2}$ at least for periods where only low-amplitude chirping single$n$ mode activity is observed, as during the $60 \mathrm{~ms}$ time window analyzed here.

Of course, from the original JT-60U experiments, we know that the present scenario is subject relaxation events known as abrupt large-amplitude events (ALE) [6], which cause significant abrupt drops in the fast ion density at intervals of about 40-60 ms [7, 8]. Reduced modeling of the impact of ALEs on the fast ion profiles and (subsequently) on their velocity distribution will probably require a more sophisticated technique than the simple profile smoothing procedure suggested above. Before that, we need to develop a better physical understanding of ALEs.

The success of the present benchmark study paves the way for applying the multi-phase method to the simulation of ALEs and the study of the underlying trigger mechanisms. Since ALEs last less than $1 \mathrm{~ms}$, the multiphase method employed here should be able to capture them, provided that the hybrid model contains all essential physics ingredients. Indeed, significant progress

\footnotetext{
${ }^{2}$ On the Helios supercomputer, the classical simulation using 256 cores took only 1-2 days for $60 \mathrm{~ms}$. In order to reduce communication overhead, that simulation was parallelized only over particles. In contrast, the multi-phase and hybrid simulations discussed above were parallelized on 4096 cores using spatial domain decomposition (cf. Table 1).
} 
has recently been made with the simulation of ALEs in JT-60U plasmas using both multi-phase and hybrid simulations [18] and the results will be published elsewhere in the near future.

\section{Acknowledgments}

One of the authors (A.B.) would like to thank Kouji Shinohara and the JT-60U Team for providing experimental data for this study and Nobuyuki Aiba for the reconstruction of the MHD equilibrium. This work has been partially supported by Grant-in-Aid for Scientific Research from the Japan Society for the Promotion of Science (JSPS, Grant No. 16K18341); the NIFS Collaborative Research Program (NIFS12KNTT016); and by MEXT as "Priority Issue on Post-K computer" (Accelerated Development of Innovative Clean Energy Systems). The simulations were carried out using the supercomputer system Helios at IFERC in Rokkasho/Japan, under the Broader Approach collaboration between Euratom and Japan, implemented by Fusion for Energy and QST (Project ID: EPTRANS3); and the K Computer of the RIKEN Advanced Institute for Computational Science in Kobe/Japan (Project IDs: hp160117, hp170260).

[1] I. Bernstein, J. Greene, M. Kruskal, Exact nonlinear plasma oscillations, Phys. Rev. 108 (3) (1957) 546.

[2] T. O'Neil, Collisionless damping of nonlinear plasma oscillations, Phys. Fluids 8 (12) (1965) 2255.

[3] H. Berk, B. Breizman, N. Petviashvili, Line broadened quasilinear burst model [fusion plasma], Nucl. Fusion 35 (12) (1995) 1661.

[4] A. Bierwage, K. Shinohara, Y. Todo, N. Aiba, M. Ishikawa, G. Matsunaga, M. Takechi, M. Yagi, Self-consistent long-time simulation of chirping and beating energetic particle modes in JT-60U plasmas, Nucl. Fusion 57 (1) (2017) 0160036.

[5] L. Chen, Theory of magnetohydrodynamic instabilities excited by energetic particles in tokamaks, Phys. Plasmas 1 (5) (1994) 1519.

[6] Y. Kusama, G. Kramer, H. Kimura, M. Saigusa, T. Ozeki, K. Tobita, T. Oikawa, K. Shinohara, T. Kondoh, M. Moriyama et al., Characteristics of Alfvén eigenmodes, burst modes and chirping modes in the Alfvén frequency range driven by negative ion based neutral beam injection in JT-60U, Nucl. Fusion 39 (11Y) (1999) 1837.

[7] K. Shinohara, M. Takechi, M. Ishikawa, Y. Kusama, K. Tsuzuki, K. Urata, H. Kawashima, K. Tobita, A. Fukuyama, C. Cheng et al, Energetic particle physics in JT-60U and JFT-2M, Plasma Phys. Control. Fusion 46 (7) (2004) S31.

[8] M. Ishikawa, M. Takechi, K. Shinohara, Y. Kusama, C. Cheng, G. Matsunaga, Y. Todo, N. Gorelenkov, G. Kramer, R. Nazikian et al., Energetic ion transport by abrupt large-amplitude events induced by negative-ion-based neutral beam injection in the JT60U, Nucl. Fusion 45 (12) (2005) 1474.

[9] Y. Todo, M. V. Zeeland, A. Bierwage, W. Heidbrink, Multiphase simulation of fast ion profile flattening due to Alfvén eigenmodes in a DIII-D experiment, Nucl. Fusion 54 (2014) 104012.
[10] Y. Todo, T. Sato, Linear and nonlinear particlemagnetohydrodynamic simulations of the toroidal Alfvén eigenmode, Phys. Plasmas 5 (5) (1998) 1321.

[11] Y. Todo, K. Shinohara, M. Takechi, M. Ishikawa, Nonlocal energetic particle mode in a JT-60U plasma, Phys. Plasmas 12 (1) (2005) 012503.

[12] Y. Todo, Properties of energetic-particle continuum modes destabilized by energetic ions with beam-like velocity distributions, Phys. Plasmas 13 (2006) 082503.

[13] Y. Todo, M. V. Zeeland, A. Bierwage, W. Heidbrink, M. Austin, Validation of comprehensive magnetohydrodynamic hybrid simulations for Alfvén eigenmode induced energetic particle transport in DIII-D plasmas, Nucl. Fusion 55 (7) (2015) 073020.

[14] Y. Todo, M. V. Zeeland, W. Heidbrink, Fast ion profile stiffness due to the resonance overlap of multiple Alfvén eigenmodes, Nucl. Fusion 56 (11) (2016) 112008.

[15] A. Bierwage, K. Shinohara, Orbit-based analysis of resonant excitations of Alfvén waves in tokamaks, Phys. Plasmas 21 (11) (2014) 112116.

[16] E. by JAEA Committee on the Usage of IFERC-CSC, IFERC Project Team and IFERC-CSC Home Team, Progress report of Japanese simulation research projects using the highperformance computer system Helios in the International Fusion Energy Research Centre, J. Plasma Fusion Res. 92 (3) (2016) 157.

[17] A. Bierwage, Y. Todo, N. Aiba, K. Shinohara, Sensitivity study for N-NB-driven modes in JT-60U: Boundary, diffusion, gyroaverage, compressibility, Nucl. Fusion 56 (10) (2016) 106009.

[18] A. Bierwage, K. Shinohara, Y. Todo, N. Aiba, M. Ishikawa, G. Matsunaga, M. Takechi, M. Toma, M. Yagi, Firstprinciple simulations reproduce multiple cycles of abrupt large relaxation events in beam-driven JT-60U plasmas, oral $\mathrm{TH} / 4-3$, in: Proceedings of the 26th Fusion Energy Conference, Oct 17-22, 2016, Kyoto, Japan, edited by International Atomic Energy Agency (IAEA), Vienna, 2016, https://nucleus.iaea.org/sites/fusionportal/Pages/Fusion 